\title{
Modelling formation of a drug reservoir in the stratum corneum and its impact on drug monitoring using reverse iontophoresis
}

\author{
Yvonne Paulley $^{\mathrm{a}}$, M. Begoña Delgado-Charro ${ }^{\mathrm{a}, \mathrm{b}}$ and K.A. Jane White ${ }^{\mathrm{a}, \mathrm{c} *}$ \\ ${ }^{a}$ Centre for Mathematical Biology, University of Bath, Bath BA2 7AY, UK; ${ }^{b}$ Department of \\ Pharmacy and Pharmacology, University of Bath, Bath BA2 7AY, UK; ${ }^{c}$ Department of Mathematical \\ Sciences, University of Bath, Bath BA2 7AY, UK
}

(Received 18 January 2010; final version received 28 May 2010)

\begin{abstract}
Reverse iontophoresis is a relatively new technique for non-invasive drug monitoring in the body. It involves a small electrical current being passed through the skin to facilitate the movement of small charged ions and polar molecules on the skin's surface where the amount of drug can then be measured and hence an accurate estimate of the blood concentration can be made. In vivo studies for several molecules show that initially large amounts of drug are extracted from the body, which are unrelated to the magnitude of the blood concentration; over time the fluxes of extraction decrease to a level proportional to the steady state blood concentration. This suggests that, at first, the drug is being extracted from some source other than the blood; one such candidate for this source is the dead cells which form the stratum corneum. In this paper, we construct two related mathematical models; the first describes the formation of the drug reservoir in the stratum corneum as a consequence of repeated drug intake and natural death of skin cells in the body. The output from this model provides initial conditions for the model of reverse iontophoresis in which charged ions from both the blood and the stratum corneum reservoir compete for the electric current. Model parameters are estimated from data collected for lithium monitoring. Our models will improve interpretation of reverse iontophoretic data by discriminating the subdermal from the skin contribution to the fluxes of extraction. They also suggest that analysis of the skin reservoir might be a valuable tool to investigate patients' exposure to chemicals including therapeutic drugs.
\end{abstract}

Keywords: reverse iontophoresis; non-invasive sampling; drug monitoring; drug reservoir; mathematical model; lithium

\section{Introduction}

Reverse iontophoresis is a relatively new technique for non-invasive drug monitoring in the body. It involves a small electrical current being passed through the skin to facilitate the movement of small charged ions and polar molecules to the skin's surface where the amount of drug can then be measured and hence an accurate estimate of the blood concentration can be made. Figure 1 is a schematic of this process. An electric current is applied to the surface of the skin. The current density must be maintained at less than $0.5 \mathrm{~mA} \mathrm{~cm}^{-2}$ in order to prevent skin damage [19]. The reduction and oxidation reactions occurring at the electrodes provoke a charge imbalance at the anodal and cathodal solution; as a consequence ions flow across the skin to keep electroneutrality [18].

*Corresponding author. Email: maskajw@bath.ac.uk 


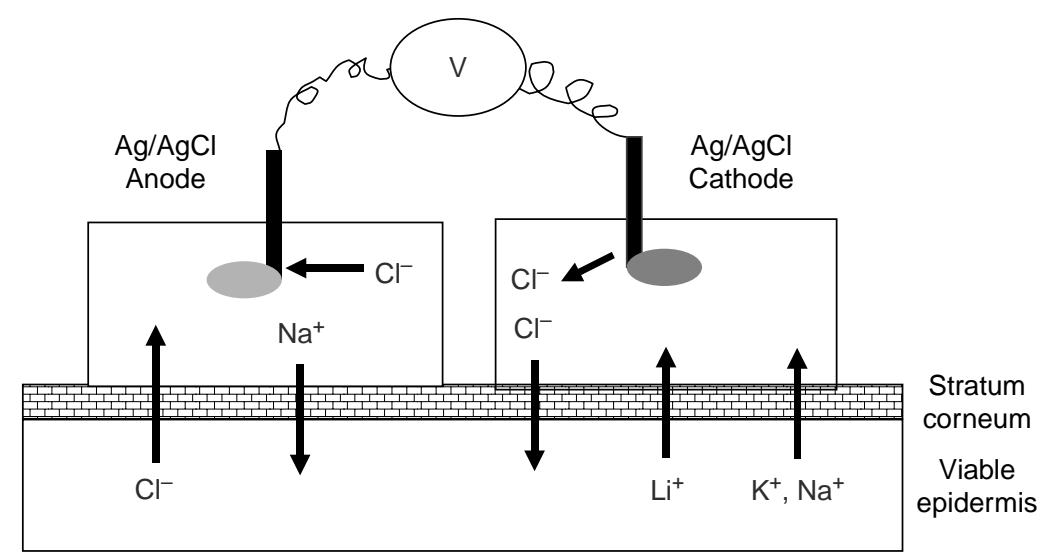

Figure 1. Schematic of the process of reverse iontophoresis reproduced from [18].

This results in positive ions being drawn to the cathode and negative ions to the anode. This process is known as electromigration and is the focus of our work here.

Two other transport mechanisms may be involved in the movement of charged ions in the skin:

1. Electroosmosis. This is caused by the natural negative charge of the skin [18]; when an electric charge is applied, a solvent flow occurs in the anode to the cathode direction. This solvent flow allows transporting neutral and zwiterrionic species and adds to the transport of cations by electromigration.

2. Passive diffusion. This occurs regardless of whether the electric current is running and its importance as a transport method appears to vary depending on the molecule in question.

It has been shown that there is a linear correlation for any substance extracted between the amount of drug collected outside the skin and that present in the subdermal concentration [9]. However, electromigration is not ion-specific: any free ion sufficiently mobile and concentrated will move in order to maintain neutrality. This ion competition for charge carrying is expressed via the transport number or fraction of the total charge transported by a specific ion. Lithium is an excellent candidate to be sampled via transdermal iontophoresis as recently shown [16] because it is a small and charged drug whose therapeutic concentrations are relatively high. Nevertheless, lithium ions compete for charge carrying with other cations present subdermally. This 'competition' between ions to carry the charge across the skin barrier, must be modelled when trying to predict the amount of drug collected at the pad.

Three potential pathways could be followed by ions moving as a result of electromigration: the transcellular route (through the corneocytes of the stratum corneum), the paracellular route (through the lipid bilayer which holds the cells together) and along the appendages of the skin (such as hair follicles and sweat glands). Of the three, the appendageal route appears to be the most important during reverse iontophoresis (as demonstrated by Cullander and Guy [5]) as it provides the least resistance to the movement of ions.

The time of each extraction period must be sufficiently long to ensure that enough analyte is detectable with the current analytic chemistry methods available, but not so long that significant changes in the serum concentration have occurred. This implies that 
reverse iontophoresis can only provide an estimation of the average level of drug in the body during the monitoring period. Some compounds fluctuate in the body very rapidly and so reverse iontophoresis is currently not a useful technique for monitoring such chemicals. However, the technique is ideal for drugs at 'steady-state' as a prolonged sampling time will still give accurate results for the average amount of drug in the body.

In this paper, lithium has been chosen as the focus molecule to extract via reverse iontophoresis. It is a small, positively charged ion which is prescribed to bipolar sufferers as the best method to stabilize moods and prevent the likelihood of suicide. However, it can be toxic at high concentrations and, therefore, its presence in the body must be carefully monitored, as well as in keeping the concentration within the therapeutic window $(0.4-1.4 \mathrm{mEq} / \mathrm{l}[33])$. Poor compliance with the specified drug dosage regimen is frequent among patients [29] and, therefore, it would be beneficial to have a method to test patients' adherence to their therapy. Blood levels have the disadvantage that they only correspond to the amount of lithium taken on that day.

Lithium has additional advantages: it does not bind to protein and therefore exists in the body purely as free cations which are sampled via reverse iontophoresis efficiently. It also distributes evenly in the total body water space, and is not metabolized, so its elimination is limited to renal excretion. The renal excretion of lithium varies with creatinine clearance [2], $\mathrm{pH}$ of urine [7] and dehydration as well as the time of day (night clearance is lower than daytime); however, lithium clearance stays within a range of $10-40 \mathrm{ml} / \mathrm{min}$, resulting in an elimination half-life of $12-27 \mathrm{~h} \mathrm{[2].} \mathrm{This} \mathrm{is} \mathrm{long} \mathrm{compared}$ to the average extraction period (about $2 \mathrm{~h} \mathrm{[16])} \mathrm{and} \mathrm{so} \mathrm{there} \mathrm{should} \mathrm{not} \mathrm{be} \mathrm{significant}$ changes in the blood concentration within this time.

It has also been shown [16] in vivo that the extraction fluxes of lithium, after the $30 \mathrm{~min}$ warm up period, are very closely correlated with the serum concentration. However, for several molecules (including lithium [16], lactate [24] and glucose [25]), in vivo data collected in this time shows an initially large amount of drug being extracted from the body during the 'warm up' period, which is unrelated to the magnitude of the serum concentration. Over time, the fluxes of the amount of drug decreases in a smooth curve to reach a level proportional to the steady state of the blood concentration [16]; these behaviours are shown from experimental data in Figure 2. This suggests that, at first, the drug molecule is being extracted from some other source as well as the blood.

It has been suggested that the dead cells of the stratum corneum act as a 'reservoir', where the repeated drug intake leads to a build up of ions [34]. The part of the reservoir directly underneath the collection pad must therefore be emptied before the readings correspond to the blood alone. In vitro iontophoresis experiments performed with pig skin sourced from animals unexposed to lithium so that no build up has been possible, show a slow increase of the amount of drug being extracted until the steady state concentration is displayed and thus strengthen this hypothesis [18].

While currently this reservoir is a hindrance to monitoring, it is hoped that it could hold some useful information about the patient's drug taking history. For example, it could be possible to predict if the patient had taken his/her dose regularly and at the correct intervals from the concentration of drug in the reservoir. If the initial readings from the extraction period differed from these predicted values it may suggest that the patient had been trying to avoid medication.

The purpose of this paper is to build a mathematical model which can describe the development of a drug reservoir in the stratum corneum upon repeated drug administration and hence to explore the impact of a drug reservoir on the monitoring of lithium using reverse iontophoresis. This is a novel modelling study; previously compartmental models 

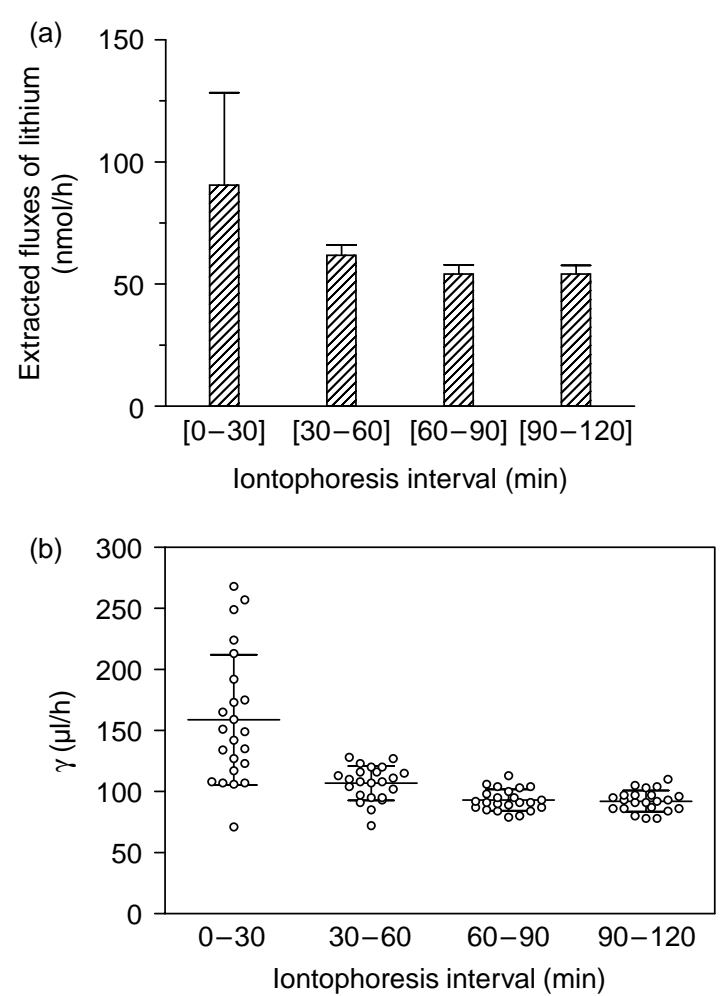

Figure 2. Reproduced from [16]. (a) Experimental data for the flux of lithium collected from in vivo patients during reverse iontophoresis drug monitoring; (b) ratio of lithium flux collected to serum concentration of lithium $(\gamma)$ as a function of duration of iontophoresis.

have been developed to explore iontophoretic delivery of drugs [14,15]. These models incorporate a delay in transversing the stratum corneum but do not account for drug loss through desquamation of the stratum corneum nor the ionic competition for charge. By contrast, [12,13] incorporate ionic competition in a model of iontophoresis but they are only interested in how ions move across the stratum corneum. Here we reverse the direction of interest by considering reverse iontophoresis, we model the formation of a drug reservoir in the stratum corneum and we add in the essential ionic competition. In order to obtain quantitative results to compare to real data, the findings shall be restricted to values for lithium monitoring - however, the basis of the model should stand for other ions, for example lactate which is monitored in critical care patients, athletes and shock patients, as well as being studied in the cosmetic industry. It is hoped that the ability to draw conclusions from these readings would make the iontophoresis devices currently on the market much more informative.

\section{Mathematical modelling}

We separate the modelling problem into two stages to reflect the very different timescales which are being considered. The first model considers the formation of a drug reservoir in the stratum corneum and has a timescale of the order of weeks. The second model determines how iontophoresis draws the drug from the reservoir and blood compartments 
using a timescale of the order of hours. The models are linked since output from the first model provides initial conditions for the second.

\subsection{Model 1: Formation of the drug reservoir}

This model has two compartments, for the amount of lithium in the body $\left(x_{1}(t)\right)$ and stratum corneum reservoir $\left(x_{2}(t)\right)$ respectively, as shown in Figure 3(a). By using this model structure, we are assuming that:

1. Both compartments are homogeneous and well mixed [27].

2. Any changes that occur in the blood serum drug levels reflect changes occurring in tissue drug levels [8]. Blood serum is the same as blood plasma without various clotting factors; it is general practice to use blood serum instead of blood values, as taking whole blood measurements can cause interference in several blood assay methods [33].

3. Blood serum is in equilibrium with both the blood and living cells in the body.

We assume that the drug is administered in equal amounts daily. Taking a constant absorption rate $k$, the rate at which the drug reaches the serum compartment is

$$
r(t)=\sum_{i=1}^{N} \delta k \exp \left(-k\left(t-T_{i}\right)\right) .
$$

where $\delta$ is the daily drug dosage, $T_{i}$ is the time at which these doses have been taken and $N$ is the number of doses which have been taken up to the present time [20]. Since lithium is almost entirely absorbed from the stomach into the blood, the full amount of drug administered will eventually be available in the serum [35]. While lithium pharmacokinetics are usually described by means of a two-compartmental model, the

(a)

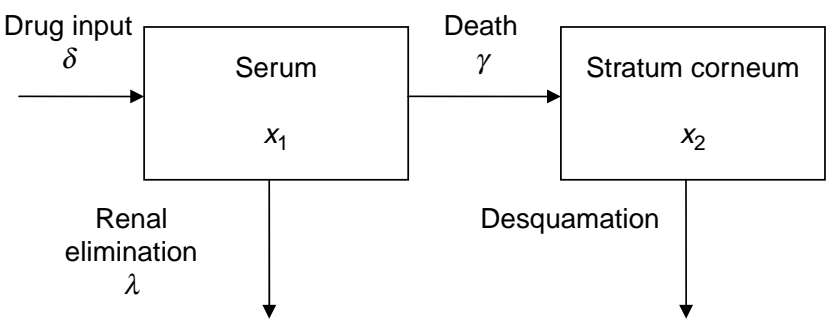

(b)

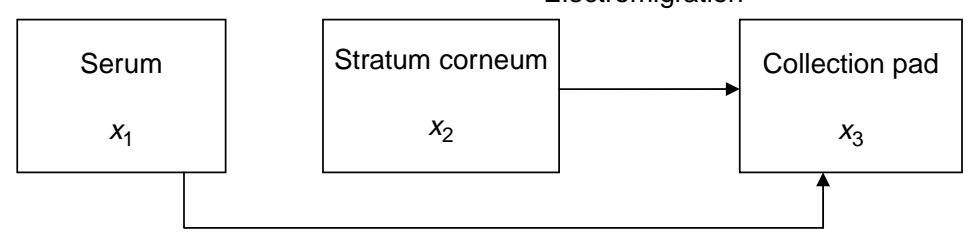

Electromigration

Figure 3. (a) Schematic of the compartmental structure used to model the formation of the drug reservoir in the stratum corneum for a patient receiving lithium treatment. (b) Schematic of the compartmental model used to model the process of reverse iontophoresis. 
drug distribution kinetics were not included in the model for two reasons: (a) the extracellular space where iontophoretic sampling takes place is part of the central compartment constituted by plasma and fast equilibrating tissues, and (b) the patients participating in the in vivo study had been receiving lithium for a sufficiently long time for the equilibrium of distribution to be achieved [2]. We assume that renal elimination rate is essentially constant and denote this constant $\lambda$.

We assume that the drug enters the stratum corneum at the rate at which living cells die to form the stratum corneum, and is then trapped in the reservoir. It is also assumed the drug is only released from the body when the outer layer of skin is shed in desquamation. In normal skin, the entire stratum corneum is shed approximately every 2 weeks [26]. In this 2-week period, the dead cells slowly travel closer to the surface of the skin. Therefore, our model is set up so that the amount of lithium entering the stratum corneum on any given day is then lost 2 weeks later; if lithium enters the stratum corneum at a rate $\gamma x_{1}(t)$, where $t$ is measured in days, the rate at which it leaves is $\gamma x_{1}(t-14)$, and $x_{1}=0$ if $t-14<0$ where $\gamma$ is the rate constant. We assume that no lithium is present in either the serum or the stratum corneum before the first dose.

Combining the above assumptions we obtain the model:

$$
\begin{gathered}
\frac{\mathrm{d} x_{1}}{\mathrm{~d} t}(t)=-\lambda x_{1}(t)-\gamma x_{1}(t)+\sum_{i=1}^{N} \delta k \exp \left(-k\left(t-T_{i}\right)\right), \\
\frac{\mathrm{d} x_{2}}{\mathrm{~d} t}(t)=\left\{\begin{array}{cc}
\gamma x_{1}(t) & t \leq 14, \\
\gamma x_{1}(t)-\gamma x_{1}(t-14) & t>14,
\end{array}\right.
\end{gathered}
$$

with initial conditions

$$
\begin{aligned}
& x_{1}(i)=0 \quad \forall i \in[-14,0], \\
& x_{2}(0)=0 .
\end{aligned}
$$

Parameter ranges and estimates, given in Table 1 are derived from published sources in Appendix A.

\subsection{Model 2: Reverse iontophoresis model}

To incorporate the effects of reverse iontophoresis we extend model 1 to include a compartment for the collection of lithium as shown in Figure 3(b). The entire skin barrier can again be modelled as a single compartment as the stratum corneum provides the limit for the rate of drug transport across the skin; all other barriers can be considered simply by using parameters corresponding to this outer layer. We assume that during the monitoring

Table 1. Parameter estimates used with model 1.

\begin{tabular}{llcc}
\hline Parameter & Definition & Value & Source \\
\hline$\delta$ & Daily dosage & $24.3 \mathrm{mmol}^{-1}$ & {$[31]$} \\
$\lambda$ & Rate of renal elimination & 0.654 days $^{-1}$ & {$[31]$} \\
$\gamma$ & Rate of lithium movement into stratum corneum & 0.005 days $^{-1}$ & {$[6,27]$} \\
$k$ & Lithium absorption rate & 4.62 days $^{-1}$ & {$[31]$} \\
\hline
\end{tabular}


period, the rate of decay of lithium within the body is small enough to be negligible, and hence the body can remain as a single compartment with a fixed concentration of lithium.

In this model, one pathway goes from the stratum corneum to the collection pad and a second from the serum to the pad. In practice the drug has to pass through the stratum corneum on its way from the serum, but this will be taken into account in the parameters used for the iontophoretic flux. The pathway through the stratum corneum is not explicitly specified: the majority of lithium may be travelling through hair follicles, but some may take the transcellular route. Again, by estimating parameters from published experimental data we do not need to specify further detail. Lithium is also excreted in sweat so it is assumed that the extraction area is cleaned to remove any residue before the current is switched on as described in Ref. [16].

As lithium is a small positively charged ion, electromigration is the primary mode of transport during iontophoresis. Previous work [18] has shown the passive diffusion and electroosmotic contributions to be negligible compared to electromigration and in consequence these minor mechanisms of transport will not be included in the model. However, small charged ions similar to lithium - namely sodium, potassium and chloride are included as they are the most abundant competitors with lithium and their concentration and mobility will have a significant impact on lithium flux.

The flux of ions of type $i(i=\mathrm{Li}, \mathrm{Na}, \mathrm{K}, \mathrm{Cl})$ across the stratum corneum can be derived analytically, from Faraday's Law $[12,13,17,18]$, to be

$$
J_{i}=\frac{I t_{i}}{F z_{i}},
$$

where $I$ is the intensity of the current, $t_{i}$ and $z_{i}$ are the transport number and valence of the type $i$ ion, respectively, and $F$ is Faraday's constant (see Table 2).

The transport number $t_{i}$ is a measure of the competition between ions for transporting the charge; it gives the fraction of charge obtained by ions of type $i$. Assuming that we have $\mathrm{Li}^{+}, \mathrm{Na}^{+}, \mathrm{K}^{+}, \mathrm{Cl}^{-}$ions, this gives

$$
t_{\mathrm{Li}}=\frac{c_{\mathrm{Li}} z_{\mathrm{Li}} u_{\mathrm{Li}}}{c_{\mathrm{Li}}\left|z_{\mathrm{Li}}\right| u_{\mathrm{Li}}+c_{\mathrm{Na}}\left|z_{\mathrm{Na}}\right| u_{\mathrm{Na}}+c_{\mathrm{K}}\left|z_{\mathrm{K}}\right| u_{\mathrm{K}}+c_{\mathrm{Cl}}\left|z_{\mathrm{Cl}}\right| u_{\mathrm{Cl}}}
$$

as the transport number for lithium, where $u_{i}$ is the aqueous mobility of the $i$ th ion in $\mathrm{cm}^{2} \mathrm{~s}^{-1} \mathrm{~V}^{-1}$ (assumed to be constant in all compartments), $c_{i}$ is the concentration in

Table 2. Parameter estimates used with model 2.

\begin{tabular}{llcc}
\hline Parameter & Definition & Value & Source \\
\hline$u_{\mathrm{Li}}$ & Aqueous mobility of lithium & $4.01 \times 10^{-4} \mathrm{~cm}^{2} \mathrm{~s}^{-1} \mathrm{~V}^{-1}$ & {$[3]$} \\
$u_{\mathrm{Na}}$ & Aqueous mobility of sodium & $5.19 \times 10^{-4} \mathrm{~cm}^{2} \mathrm{~s}^{-1} \mathrm{~V}^{-1}$ & {$[3]$} \\
$u_{\mathrm{K}}$ & Aqueous mobility of potassium & $7.62 \times 10^{-4} \mathrm{~cm}^{2} \mathrm{~s}^{-1} \mathrm{~V}^{-1}$ & {$[3]$} \\
$u_{\mathrm{Cl}}$ & Aqueous mobility of chloride & $7.91 \times 10^{-4} \mathrm{~cm}^{2} \mathrm{~s}^{-1} \mathrm{~V}^{-1}$ & {$[22]$} \\
$I$ & Intensity of current & $0.8 \mathrm{~mA}$ & {$[16]$} \\
$F$ & Faraday's constant & $96.487 \mathrm{Cmmol}$ & {$[16]$} \\
$S_{\mathrm{Na}}$ & Amount of sodium ions in the serum & $5838 \mathrm{mmol}$ & {$[16]$} \\
$S_{\mathrm{K}}$ & Amount of potassium ions in the serum & $155.4 \mathrm{mmol}$ & {$[16]$} \\
$S_{\mathrm{Cl}}$ & Amount of chloride ions in the serum & $4200 \mathrm{mmol}$ & {$[35]$} \\
$x_{\mathrm{Na}}$ & Amount of available sodium ions in the SC & $0.2688 \mathrm{mmol}$ & {$[11]$} \\
$x_{\mathrm{K}}$ & Amount of available potassium ions in the SC & $0.5056 \mathrm{mmol}$ & {$[11]$} \\
$x_{\mathrm{Cl}}$ & Amount of available chloride ions in the SC & $0.7488 \mathrm{mmol}$ & {$[11]$} \\
\hline
\end{tabular}


the compartment and $z_{i}$ is the valence ( +1 for lithium, sodium, potassium and -1 for chloride). To express the transport number in terms of amounts, we can use the transformation

$$
c_{i} \times V_{\mathrm{d}}=x_{i},
$$

where $V_{\mathrm{d}}$ is the volume of distribution of the particular compartment with respect to ion $i$. For the serum compartment it may be expected that this value would be different for the different competitors. However, as all the ions in question are small and hydrophilic they can be assumed to distribute to similar volumes of distribution - lithium distributes nearly evenly in the total body water space [2] so it can be assumed that $V_{\mathrm{d}} \cong 42 L$.

The problem in the stratum corneum is slightly less well-defined as it is not known where the lithium is stored in the reservoir with respect to cells, follicles, etc. However, it is sensible to assume that all competitors are extracted from the same location in the reservoir, and again as all the competitors are similarly charged it is assumed that they have similar volumes of distribution within the reservoir. Hence, for both compartments, the concentrations can be converted to amounts as the volume term will cancel from the numerator and denominator of (3). The transport number for lithium is therefore given as

$$
t_{\mathrm{Li}}=\frac{x_{\mathrm{Li}} u_{\mathrm{Li}}}{x_{\mathrm{Li}} u_{\mathrm{Li}}+x_{\mathrm{Na}} u_{\mathrm{Na}}+x_{\mathrm{K}} u_{\mathrm{K}}+x_{\mathrm{Cl}} u_{\mathrm{Cl}}} .
$$

We hold the levels of sodium, potassium and chloride ions in both compartments constant over the period of ion extraction since they are found in much higher levels than lithium. We also assume that the amount of lithium in the serum is constant, so that $x_{1}(t)=F_{1}$, calculated as the final level of lithium at the end of the simulation from model 1 (for our purposes this is the value $x_{1}(30)$ ).

Combining these modelling assumptions, the decrease in the amount of $\mathrm{Li}^{+}$in the stratum corneum as a result of reverse iontophoresis is governed by

$$
\frac{\mathrm{d} x_{2}}{\mathrm{~d} t}=-\frac{I}{F} \frac{x_{2} u_{\mathrm{Li}}}{x_{2} u_{\mathrm{Li}}+x_{\mathrm{Na}} u_{\mathrm{Na}}+x_{\mathrm{K}} u_{\mathrm{K}}+x_{\mathrm{Cl}} u_{\mathrm{Cl}}},
$$

where $x_{\mathrm{Na}}, x_{\mathrm{K}}$ and $x_{\mathrm{Cl}}$ correspond to the amounts of the competitors in the stratum corneum.

The amount of $\mathrm{Li}^{+}$collected on the pad is the sum of fluxes from the serum and the reservoir:

$$
\frac{\mathrm{d} x_{3}}{\mathrm{~d} t}=\frac{I}{F} \frac{x_{2} u_{\mathrm{Li}}}{x_{2} u_{\mathrm{Li}}+x_{\mathrm{Na}} u_{\mathrm{Na}}+x_{\mathrm{K}} u_{\mathrm{K}}+x_{\mathrm{Cl}} u_{\mathrm{Cl}}}+\frac{I}{F} \frac{F_{1} u_{\mathrm{Li}}}{F_{1} u_{\mathrm{Li}}+S_{\mathrm{Na}} u_{\mathrm{Na}}+S_{\mathrm{K}} u_{\mathrm{K}}+S_{\mathrm{Cl}} u_{\mathrm{Cl}}},
$$

where again it is assumed that the serum concentrations of the competitors $\left(S_{i}, i=\mathrm{Na}, \mathrm{K}\right.$, $\mathrm{Cl}$ ) are constant throughout the iontophoretic period.

The initial conditions are taken as

$$
\begin{aligned}
& x_{2}(0)=F_{2}, \\
& x_{3}(0)=0,
\end{aligned}
$$

where $F_{2}$ is the amount of lithium in the stratum corneum calculated from the first model as the final value attained at the end of the simulation of model 1 , scaled to include on that amount which is directly below the collection pad. This scaling was also applied to the 
other ions in the stratum corneum, since it is only those ions which directly encounter the electric current that will be collected during the relatively short extraction period. The collection pad has no maximum capacity, i.e. lithium will never be hindered from entering the pad regardless of the amounts already there. Parameter estimates used with this model are given in Table 2.

\section{Model analysis}

\subsection{Model 1: Formation of a drug reservoir}

We can solve (1a) and (1b) exactly to obtain

$$
\begin{aligned}
& x_{1}(t)=\frac{\delta k}{\lambda+\gamma-k}\left[\mathrm{e}^{-k t} \sum_{i=1}^{N} \mathrm{e}^{k T_{i}}-\mathrm{e}^{-(\lambda+\gamma) t} \sum_{i=1}^{N} \mathrm{e}^{(\lambda+\gamma) T_{i}}\right], \\
& x_{2}(t)=\left\{\begin{array}{c}
\frac{\gamma \delta k}{\lambda+\gamma-k}\left[\frac{1}{k}\left(Y-\mathrm{e}^{-k t} \sum_{i=0}^{\mathrm{FL}} \mathrm{e}^{k i}\right)+\frac{1}{\lambda+\gamma}\left(\mathrm{e}^{-(\lambda+\gamma) t} \sum_{i=0}^{\mathrm{FL}} \mathrm{e}^{(\lambda+\gamma) i}-Y\right)\right] \\
t \leq 14, \\
\frac{\gamma \delta k}{\lambda+\gamma-k}\left[\sum_{i=0}^{T}\left(\frac{-\mathrm{e}^{-k(t-i)}}{k}+\frac{\mathrm{e}^{-(\lambda+\gamma)(t-i)}}{\lambda+\gamma}\right)\right. \\
\left.+\sum_{i=0}^{T-14}\left(\frac{\mathrm{e}^{-k(t-14-i)}}{k}-\frac{\mathrm{e}^{-(\lambda+\gamma)(t-14-i)}}{\lambda+\gamma}\right)+\frac{14}{k}-\frac{14}{\lambda+\gamma}\right] \quad t>14 .
\end{array}\right.
\end{aligned}
$$

Details of this calculation are given in Appendix B. This solution gives the amount of lithium in the plasma $\left(x_{1}(t)\right)$ and stratum corneum $\left(x_{2}(t)\right)$ in a patient taking lithium daily.

Given the pharmacokinetic properties of lithium [2], it is expected that the steady state will be achieved after a few days of drug administration; therefore, the patients participating in the in vivo study will have steady state serum amounts which are wellrepresented in our model solution (see Figure 4(a)). The daily dose takes a small amount of time to enter the serum due to the absorption rate, and then the renal excretion term begins to dominate so that the amount of lithium decreases exponentially until the next dosage. The toxicity bound for lithium is at $1.5 \mathrm{mmol} / 1$ [28] which, if the total body water is taken as 421 (as quoted for a $70 \mathrm{~kg}$ human in Ref. [30]), corresponds to $63 \mathrm{mmol}$ in the body at any time. The graph produced shows a daily variation which stays below the toxic level. Moreover, serum concentration has been shown to fluctuate between 0.8 and $1.2 \mathrm{mmol} / \mathrm{l}$ on a daily basis [10], assuming a single dose taken each day. This relates to serum amounts of $33-50 \mathrm{mmol}$ which corresponds well to the results in Figure 4(a). The amount of lithium in the reservoir is shown in Figure 4(b). Initially it increases rapidly, followed by a steady increase for the 2 weeks before any lithium begins to be lost from the outer layers of the stratum corneum by desquamation. Once desquamation starts to play a role, the amount of lithium in the reservoir settles to a steady state.

\subsubsection{Non-compliance}

We use our solution to explore non-compliance, i.e. patients not taking their daily dose; the results are presented in Figure 5. There are several interesting observations which can be made: firstly there is a difference in the length of impact of non-compliance between 

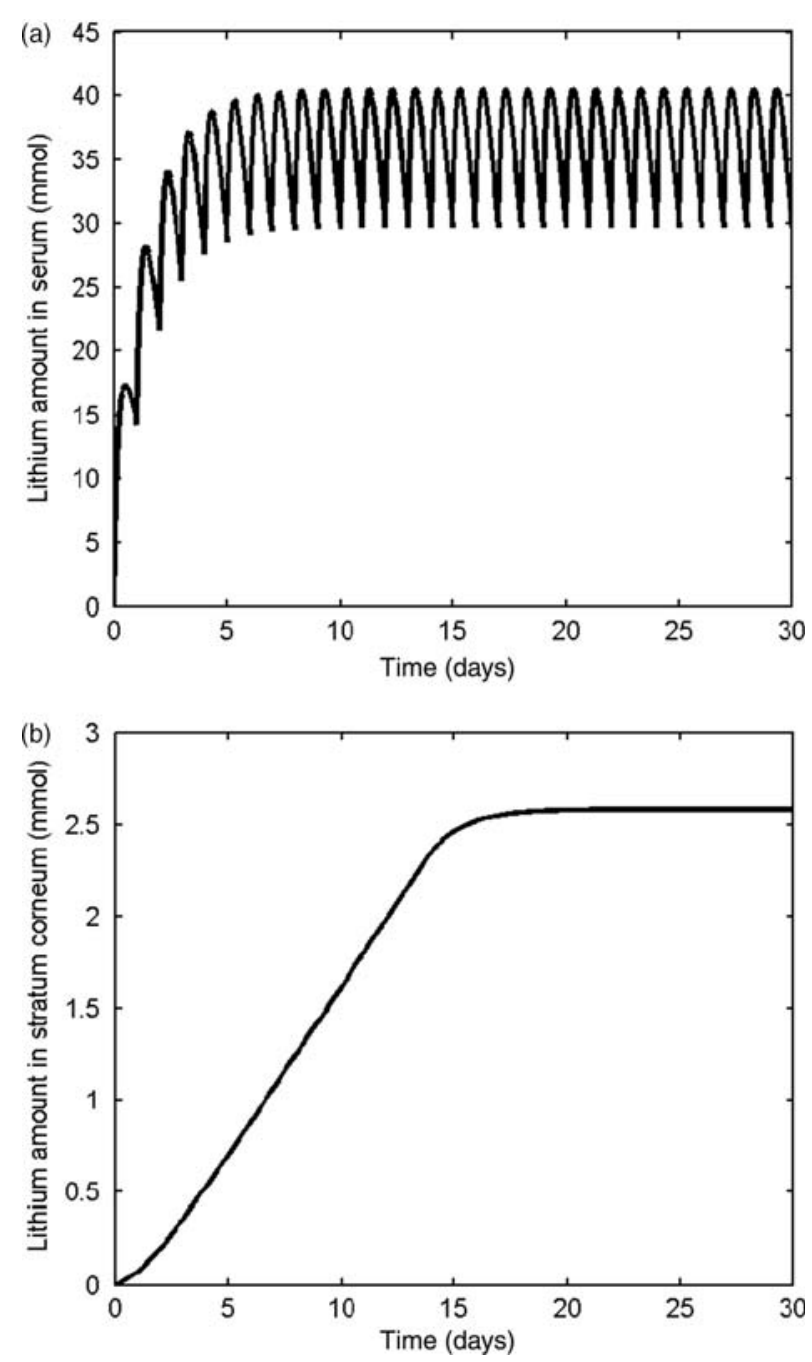

Figure 4. (a) Analytical solution for the amount of lithium in the blood serum of a naïve patient receiving daily doses of the drug. Parameter values as given in Table 1. (b) Analytical solution of the amount of lithium collected in the stratum corneum reservoir for a naïve patient receiving daily doses of the drug. Parameter values as given in Table 1.

drug levels in the serum and corresponding levels in the stratum corneum. In the serum (Figure 5(a),(c),(e)), the impact of non-compliance is short-lived and within a few days lithium levels are fully recovered. However, in the reservoir the impact is significantly longer lived (Figure 5(b),(d),(f)) and differences in lithium levels will be observable for 2 weeks following the missed dose (corresponding to the desquamation time). Moreover, if non-compliance occurs in the first 15 days of prescription for a new patient, it is unlikely to be noticed by studying drug levels in the stratum corneum reservoir; this contrasts with non-compliance in patients who have been taking the drug for a longer period of time (cf. Figure 5(b) with Figure 5(d)). Having said that, if there is only a single non-compliance event, then the actual change in lithium levels in the stratum corneum 

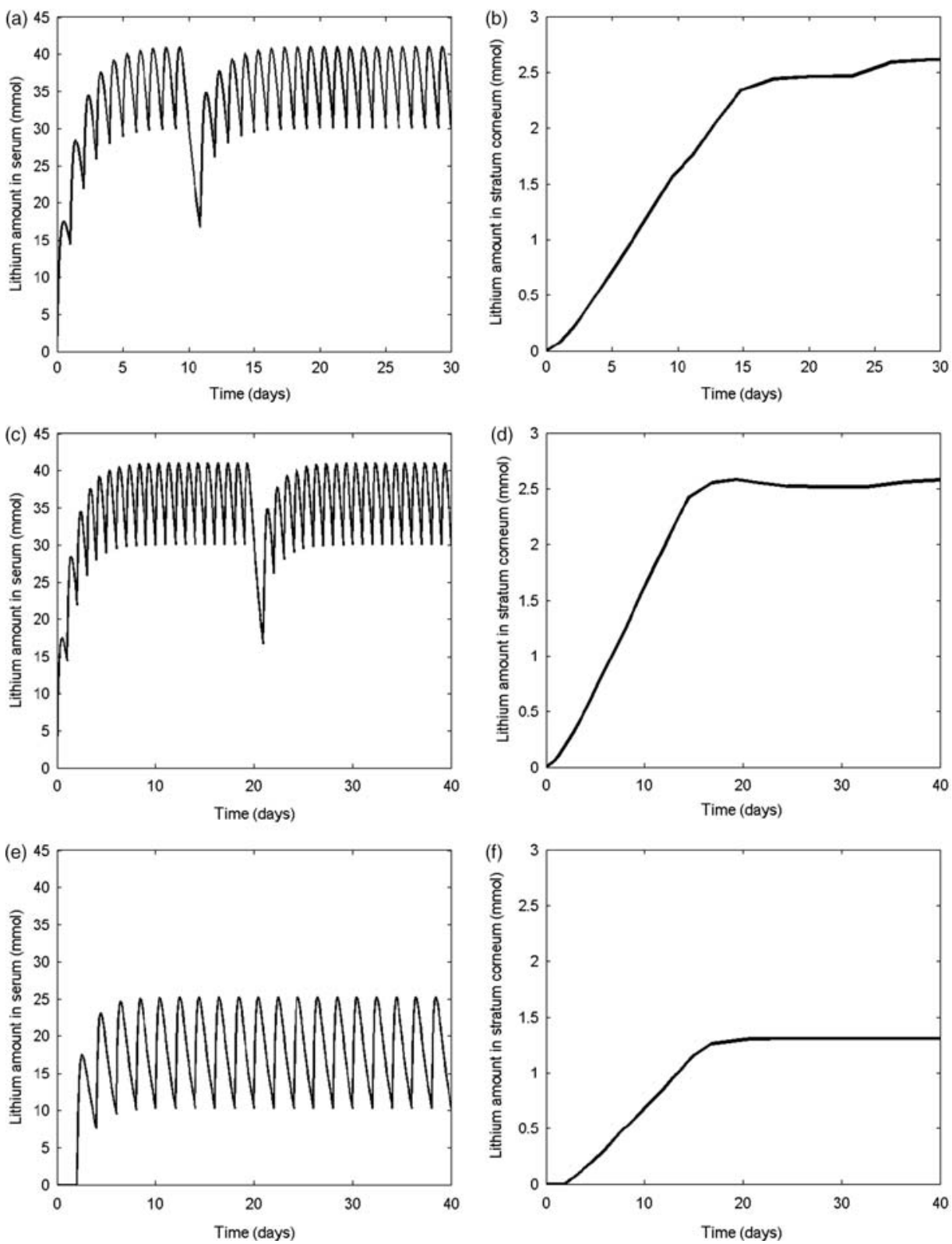

Figure 5. Effect of non-compliance with a drug regime on lithium levels in blood serum. Parameter values as given in Table 1. In (a) and (b), patient fails to take drug on day 10; in (c) and (d), patient fails to take drug on day 20; in (e) and (f), patient fails to take drug every other day. (a), (c) and (e) show the level of lithium in the serum on a daily basis whilst (b), (d) and (f) show the lithium levels in the stratum corneum.

may be difficult to detect in a clinical setting. With patients that consistently fail to take their prescription, we show that the reservoir may provide important information about the degree of non-compliance. In Figure 5(e),(f), we present results for a patient who takes his/her prescription every other day and in that case, there is a clear difference in lithium 
levels both in the serum and reservoir compared with the expected levels (Figure 5(a),(b)). These findings can now be validated by in vivo studies.

\subsection{Model 2: Reverse iontophoresis model}

This model is presented in (5)-(7) with initial conditions determined from the previous model. Again we can solve the model system exactly but not explicitly (the variable $x_{2}(t)$ can only be found implicitly). Therefore we present only numerical solutions. Because we want to compare model results with the experimental data reproduced in Figure 2, we assume that the patient complied with their dosage regimen for 30 days and iontophoresis occurred for $3 \mathrm{~h}$ at the start of the 31st day. Figure 6(a),(b) shows the amount of lithium in the stratum corneum and on the pad over a period of $150 \mathrm{~min}$. Clearly, during the first $30 \mathrm{~min}$, lithium is drained from the reservoir which accounts for the high fluxes during the warm-up period. Subsequently, the flux onto the pad originates from lithium pulled from the serum across the stratum corneum and at this point, the reverse iontophoresis technique becomes an accurate monitoring technique for monitoring lithium amounts in a patient.
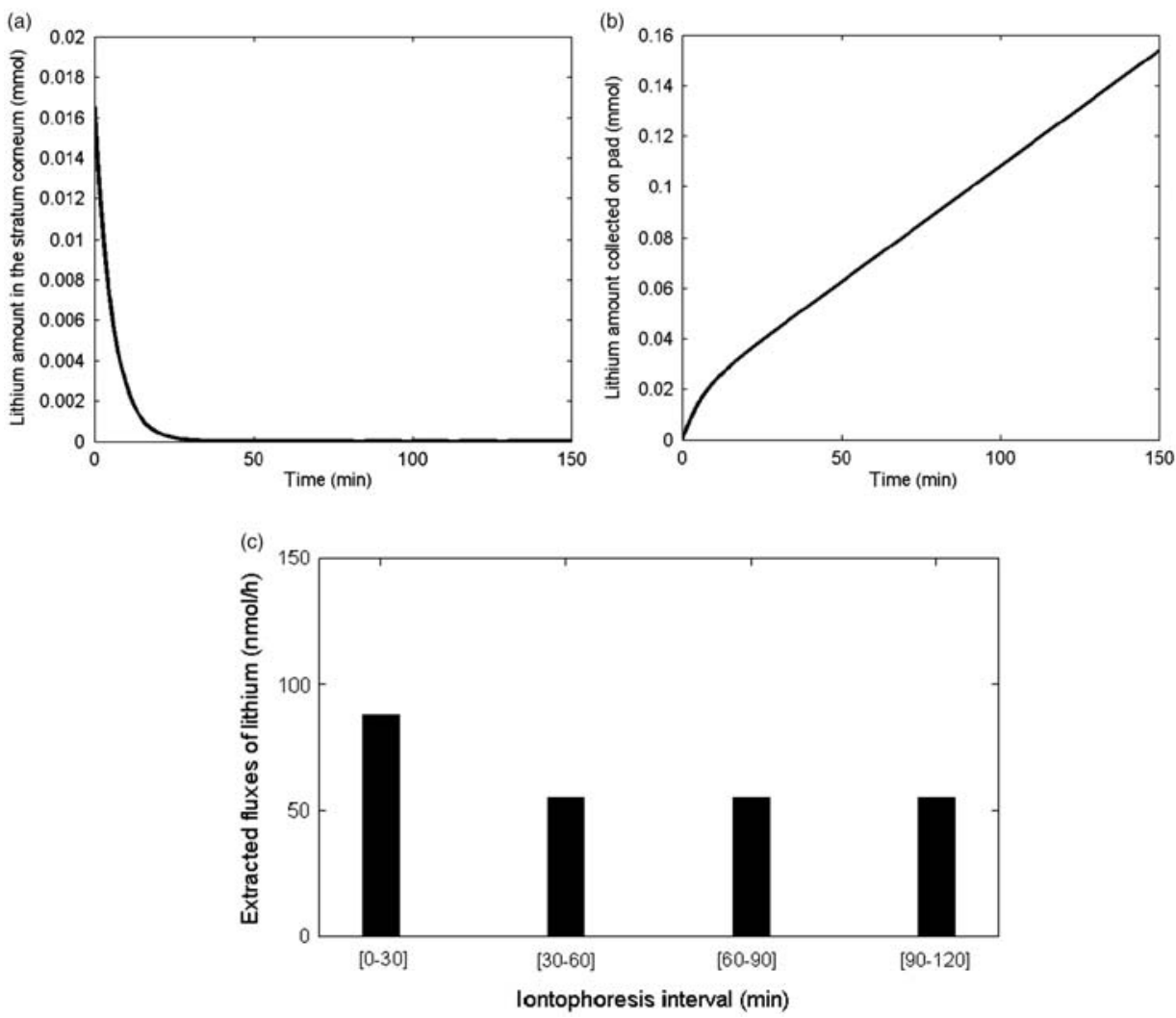

Figure 6. (a) Amount of lithium in the stratum corneum following the onset of reverse iontophoresis at time $t=0$. (b) Amount of lithium collected on the pad following the onset of reverse iontophoresis at time $t=0$. (c) Flux of lithium collected on the pad during reverse iontophoresis using parameter values as given in Tables 1 and 2 . We take $F_{1}=29.73 \mathrm{mmol}$ and $F_{2}=2.59 \mathrm{mmol}$ as determined by the final levels of lithium in the serum and stratum corneum from model 1 (shown in Figure 4(a),(b)). 
Figure 6(c) shows the flux of lithium onto the collection pad over 30 min periods. These results compare very well with the data presented in Figure 2.

\section{Discussion}

Two models have been developed to predict drug accumulation into the stratum corneum and the impact of this reservoir on reverse iontophoretic fluxes of drug extraction. These models will improve interpretation of reverse iontophoretic data by discriminating the subdermal from the skin contribution to the fluxes of extraction. They also suggest that analysis of the skin reservoir might be a valuable tool to investigate patient's exposure to chemicals including therapeutic drugs. Iontophoresis was initially developed in the 1980s [4] as a method for drug delivery, where the current accelerated the transport of molecules from outside the skin into the subcutaneous layer, but it is only in the last 10 years or so that the potential of the reverse transport was appreciated [18]. Its potential applications include the detection of diagnostic markers, drug dosage control and possibly even in determining the prognosis of a patient (for example in the case of lactate, which is produced in varying amounts when the patient is under stress [21]). The major benefit of this technique is that there should theoretically be no need for a blood sample, so pain and discomfort would be reduced, which in turn should lead to increased compliance from patients and a decreased risk of infection. Furthermore, iontophoresis devices do not require trained personnel to operate them so patients can monitor their own progress at home.

The GlucoWatch Biographer was the first reverse iontophoresis device commercialized [32]. It can return glucose levels every twenty minutes and therefore picks up changes in the blood sugar concentrations very quickly, which could prevent hyperglycaemic and hypoglycaemic events. However, reverse iontophoresis is still very much under development: an initial finger stick is needed to calibrate the machine to the blood concentration of a particular patient, and there is currently a 'warm up' period before the readings gathered relate to the steady concentration of the blood. By comparing data from in vitro and in vivo studies, the following observations about the warm up period can be made: for drug naïve patients, the period corresponds to the amount of time until lithium ions are pulled across the stratum corneum by the electric current in competition with the other charged ions being pulled; for patients who have built up a reservoir of lithium in the stratum corneum, the period corresponds to the time to empty this reservoir. Moreover, data collected during the warm up period for this second group of individuals can be used to measure non-compliance with drug regimes.

The results from our mathematical model have been able to effectively mimic the warm up period and have highlighted those parameters which play a key role in determining the level of the stratum corneum reservoir and how it is emptied during reverse iontophoresis. The model is able to estimate the degree of non-compliance for a chronically ill patient by comparing the data collected during the warm up period with the values expected for a fully compliant individual.

Using the parameter estimates presented here, the length of the warm up period was fairly consistent, around 30-60 min. To determine the key parameters driving this length of time we will extend our initial model to consider the movement of ions across the stratum corneum using a nonlinear advection-diffusion model. However, even in the absence of such a complex model, we are able to demonstrate the potential of a simple mathematical model to help in understanding how to make effective use of information contained in a skin drug reservoir both to understand patient compliance and also to guide the experimental work in parameter estimation. 


\section{References}

[1] A. Amdisen, Serum concentration and clinical supervision in monitoring of lithium treatment, Ther. Drug Monit. 2 (1980), pp. 73-83.

[2] T.L. Bettinger and M.L. Crismon, Lithium, in Applied Pharmacokinetics and Pharmacodynamics, M.E. Burton, L.M. Shaw, J.J. Shentag, and W.E. Evans, eds., 4th ed., Lippincott Williams and Wilkins, Baltimore, MD, 2006, pp. 798-812.

[3] C.M.A. Brett and A.M. Oliveira Brett, Electrochemistry: Principles, Methods, and Applications, Oxford University Press, Oxford, 1993, p. 31.

[4] R.R. Burnette, Iontophoresis, in Transdermal Drug Delivery, J. Hadgraft and R.H. Guy, eds., Marcel Dekker, New York, 1989, pp. 247-291.

[5] C. Cullander and R.H. Guy, Sites of iontophoretic current flow into the skin: Identification and characterization with the vibrating probe electrode, J. Invest. Dermatol. 97 (1992), pp. 55-64.

[6] P.M. Elias and K.R. Feingold, Skin Barrier, Taylor and Francis Group, New York, 2006.

[7] M. Gibaldi, Introduction to Biopharmaceutics, Lea and Febiger, Philadelphia, PA, 1971.

[8] M. Gibaldi and D. Perrier, Pharmacokinetics, Marcel Dekker, Inc., New York, 1975.

[9] P. Glikfield, R.S. Hinz, and R.H. Guy, Noninvasive sampling of biological fluids by iontophoresis, Pharm. Res. 6 (1989), pp. 988-990.

[10] F.K. Goodwin and K.R. Jamison, Manic-depressive Illness, Oxford University Press, New York, 1990.

[11] T.G. Grundin, G.M. Roomans, B. Forslind, M. Lindberg, and Y. Werner, X-ray microanalysis of psoriatic skin, J. Invest. Dermatol. 85 (1985), pp. 378-380.

[12] J.C. Keister and G.B. Kasting, Ionic mass transport through a homogeneous membrane in the presence of a uniform electric field, J. Memb. Sci. 29 (1986), pp. 155-167.

[13] J.C. Keister and G.B. Kasting, Application of electrodiffusion theory for a homogeneous membrane to iontophoretic transport through skin, J. Cont. Release 8 (1989), pp. 195-210.

[14] A. Kharis Nugroho, O. Della Pasqua, M. Danhof, and J.A. Bouwstra, Compartmental modelling of transdermal iontophoretic transport: I. In vitro model derivation and application, Pharm. Res. 21(11) (2004), pp. 1974-1984.

[15] A. Kharis Nugroho, O. Della Pasqua, M. Danhof, and J.A. Bouwstra, Compartmental modelling of transdermal iontophoretic transport: II. In vivo model derivation and application, Pharm. Res. 22(3) (2005), pp. 335-346.

[16] B. Leboulanger, J.-M. Aubry, G. Bondolfi, R.H. Guy, and M.B. Delgado-Charro, Lithium monitoring by reverse iontophoresis in vivo, Clin. Chem. 50(11) (2004), pp. 2091-2100.

[17] B. Leboulanger, R.H. Guy, and M.B. Delgado-Charro, Reverse iontophoresis as a non-invasive tool for lithium monitoring and pharmacokinetic profiling, Pharm. Res. 21 (2004), pp. 1214-1222.

[18] B. Leboulanger, R.H. Guy, and M.B. Delgado-Charro, Reverse iontophoresis for non-invasive transdermal monitoring, Physiol. Meas. 25 (2004), pp. R35-R50.

[19] P.W. Ledger, Skin biological issues in enhanced transdermal delivery, Adv. Drug Deliv. 9(2-3) (1992), pp. 289-307.

[20] P. Macheras and A. Iliadis, Modelling in Biopharmaceutics, Pharmacokinetics, and Pharmacodynamics: Homogeneous and Heterogeneous Approaches, Springer Science and Business Media, Inc., New York, 2006.

[21] B.A. Mizock and J.L. Falk, Lactic acidosis in critical illness, Crit. Care Med. 20 (1992), pp. 80-93.

[22] W.J. Moore, Physical Chemistry, 5th ed., Prentice Hall, Inc., London, 1972.

[23] F. Nielsen-Kudsk and A. Amdisen, Analysis of the pharmacokinetics of lithium in man, Eur. J. Clin. Pharm. 16(4) (1979), pp. 271-277.

[24] S. Nixon, A. Sieg, M.B. Delgado-Charro, and R.H. Guy, Reverse iontophoresis of L-Lactate: In vitro and in vivo studies, J. Pharm. Sci. 96(12) (2007), pp. 3457-3465.

[25] G. Rao, R.H. Guy, P. Glikfield, W.R. LaCourse, L. Leung, J. Tamada, R.O Potts and N. Azimi, Reverse iontophoresis: Non invasive glucose monitoring in humans, Pharm. Res. 12 (1995), pp. $1869-1873$.

[26] D. Roberts and R. Marks, The determination of regional and age variations in the rate of desquamation: A comparison of four techniques, J. Invest Dermatol. 74 (1980), pp. 13-16.

[27] M.S. Roberts, S.E. Cross, and Y.G. Anissimov, Factors affecting the formation of a skin reservoir for topically applied solutes, Skin Pharmacol. Physiol. 17 (2004), pp. 3-16. 
[28] A.F. Schatzberg and C.B. Nemeroff, eds., The American Psychiatric Press Textbook of Psychopharmacology, 2nd ed., American Psychiatric Press, Washington, DC, 1998.

[29] J. Scott and M. Pope, Nonadherence with mood stabilizers: Prevalence and predictors, J. Clin. Psychiatry 63 (2002), pp. 384-390.

[30] L. Shargel and A.B.C. Yu, Drug distribution and protein binding, in Applied Biopharmaceutics and Pharmacokinetics, 3rd ed., Appleton and Lange, Norwalk, CT, 1996, p. 85.

[31] D.P. Thornhill, Pharmacokinetics of ordinary and sustained-release lithium carbonate in manic patients after acute dosage, Eur. J. Clin. Pharm. 14(4) (1978), pp. 267-271.

[32] M.J. Tierney, J.A. Tamada, R.O. Popps, R.C. Eastman, K. Pitzer, N.R. Ackmann, and S.J. Fermi, The GlucoWatch biographer: A frequent automatic and noninvasive glucose monitor, Ann. Med. 32 (2002), pp. 632-641.

[33] T.N. Tozer and M. Rowland, Introduction to Pharmacokinetics and Pharmacodynamics, Lippincott Williams and Wilkins, Baltimore, MD, 2006.

[34] C.F.H. Vickers, Existence of reservoir in the stratum corneum - experimental proof, Arch. Dermatol. 88 (1963), pp. 20-33.

[35] M.E. Winter, Basic Clinical Pharmacokinetics, 4th ed., Lippincott Williams and Wilkins, Baltimore, MD, 2003.

\section{Appendix A: Estimation of parameter values}

\section{A.1 Rate of renal decay, $\lambda$}

The elimination rate can be calculated from the biological elimination half-life. This half-life is defined as the period of time it takes for the concentration of drug in the body to decrease by $50 \%$. For lithium, this can range between 12 and $27 \mathrm{~h} \mathrm{[2].} \mathrm{Clinical} \mathrm{trials} \mathrm{by} \mathrm{Thornhill} \mathrm{[31]} \mathrm{found} \mathrm{that} \mathrm{the}$ average elimination half-life for manic patients taking an acute dose of sustained-release lithium carbonate was $25.6 \mathrm{~h}$, which corresponds to 1.06 days. The elimination rate constant can thus be calculated as 0.654 by utilising the equation $\lambda=\ln 2 / t_{1 / 2}$ (assuming exponential decay).

\section{A.2 Dosage, $\delta$}

The amount to give in a single dose is in itself a complex pharmacokinetic problem as the doseresponse ratio for lithium can vary considerably between patients [1]. It is generally accepted that taking three doses per day is safest as this causes the serum concentration to vary less, although single daily doses are frequently used to facilitate compliance. In order to replicate a real system as closely as possible the value for $\delta$ was again taken from Thornhill's study [31], and hence $24.3 \mathrm{mmol}$ of sustained-release lithium was taken as the daily dosage. This seems to be a sensible value to use, as $24 \mathrm{mmol}$ was used in both single and multiple dose experiments by Nielsen-Kudsk and Amdisen [23], and the patients in the in vivo study [16] were receiving doses of $12-36 \mathrm{mmol} / \mathrm{day}$.

\section{A.3 Rate of lithium movement into the stratum corneum, $\gamma$}

The depth of the stratum corneum remains constant at about $20 \mu \mathrm{m} \mathrm{[26]} \mathrm{and} \mathrm{hence} \mathrm{the} \mathrm{rate} \mathrm{of}$ formation of the stratum corneum should equal its rate of loss. In our compartmental model, the parameter $\gamma$ is movement from the serum into the stratum corneum; since it is only the viable epidermal cells which make this transition, we scale the rate of movement by the fraction $f$ of possible lithium that could make this transition. We estimate this fraction as 0.1 . The stratum corneum takes between 14 and 28 days to renew [26] and so the average time that lithium would remain in the stratum corneum is about 20 days. Combining these considerations, we take $\gamma=1 / 200$ days $^{-1}$.

\section{A.4 Absorption rate constant, $k$}

Similarly to calculating the elimination rate constant, this can be calculated as $k=\ln 2 / t_{1 / 2}$, where $t_{1 / 2}$ is the absorption half-life of lithium and is quoted by Thornhill [31] for the sustained-release lithium as $3.73 \mathrm{~h}$ or 0.155 days. Hence $k=4.62$ days $^{-1}$. 


\section{Appendix B: Exact solution of model 1}

To solve (1a):

$$
\frac{\mathrm{d} x_{1}}{\mathrm{~d} t}(t)+(\lambda+\gamma) x_{1}(t)=\sum_{i=1}^{N} \delta k \exp \left(-k\left(t-T_{i}\right)\right)
$$

we use the integrating factor $\mathrm{e}^{(\lambda+\gamma) t}$ and solve on sequential intervals $T<t<T+1$ to give the solution

$$
x_{1}(t)=\frac{\delta k}{\lambda+\gamma-k}\left[\mathrm{e}^{-k t} \sum_{i=1}^{N} \mathrm{e}^{k T_{i}}-\mathrm{e}^{-(\lambda+\gamma) t} \sum_{i=1}^{N} \mathrm{e}^{(\lambda+\gamma) T_{i}}\right] .
$$

To find $x_{2}(t)$ we solve (1b) in two time intervals. For $t<14$,

$$
\frac{\mathrm{d} x_{2}}{\mathrm{~d} t}=\gamma x_{1}(t)=\frac{\gamma \delta k}{\lambda+\gamma-k}\left[\mathrm{e}^{-k t} \sum_{i=0}^{\mathrm{FL}} \mathrm{e}^{k i}-\mathrm{e}^{-(\lambda+\gamma) t} \sum_{i=0}^{\mathrm{FL}} \mathrm{e}^{(\lambda+\gamma) i}\right],
$$

where $\mathrm{FL}=$ floor $(t)$ is the integer part of $t$. Integrating with respect to $t$, we obtain

$$
x_{2}(t)=\frac{\gamma \delta k}{\lambda+\gamma-k}\left[\frac{-\mathrm{e}^{-k t}}{k} \sum_{i=0}^{\mathrm{FL}} \mathrm{e}^{k i}+\frac{\mathrm{e}^{-(\lambda+\gamma) t}}{\lambda+\gamma} \sum_{i=0}^{\mathrm{FL}} \mathrm{e}^{(\lambda+\gamma) i}\right]+B .
$$

The parameter $B$ is derived using the value of $x_{1}(t)$ at each integer $t$ giving for $0<t<14$

$$
x_{2}(t)=\frac{\gamma \delta k}{\lambda+\gamma-k}\left[\frac{1}{k}\left(Y-\mathrm{e}^{-k t} \sum_{i=0}^{\mathrm{FL}} \mathrm{e}^{k i}\right)+\frac{1}{\lambda+\gamma}\left(\mathrm{e}^{-(\lambda+\gamma) t} \sum_{i=0}^{\mathrm{FL}} \mathrm{e}^{(\lambda+\gamma) i}-Y\right)\right],
$$

where $Y=\operatorname{ceil}(t)$ is the nearest integer value greater than $t$.

Now when the desquamation process begins (i.e. $t>14$ ),

$$
\frac{\mathrm{d} x_{2}}{\mathrm{~d} t}=\frac{\gamma \delta k}{\lambda+\gamma-k}\left[\mathrm{e}^{-k t} \sum_{i=0}^{\mathrm{FL}} \mathrm{e}^{k i}-\mathrm{e}^{-(\lambda+\gamma) t} \sum_{i=0}^{\mathrm{FL}} \mathrm{e}^{(\lambda+\gamma) i}-\mathrm{e}^{-k(t-14)} \sum_{i=0}^{\mathrm{FL}-14} \mathrm{e}^{k i}+\mathrm{e}^{-(\lambda+\gamma)(t-14)} \sum_{i=0}^{\mathrm{FL}-14} \mathrm{e}^{(\lambda+\gamma) i}\right],
$$

which can simply be integrated with respect to $t$ to give

$$
\begin{aligned}
x_{2}(t)= & \frac{\gamma \delta k}{\lambda+\gamma-k}\left[\frac{-\mathrm{e}^{-k t}}{k} \sum_{i=0}^{T} \mathrm{e}^{k i}+\frac{\mathrm{e}^{-(\lambda+\gamma) t}}{\lambda+\gamma} \sum_{i=0}^{T} \mathrm{e}^{(\lambda+\gamma) i}\right. \\
& \left.+\frac{\mathrm{e}^{-k(t-14)}}{k} \sum_{i=0}^{T-14} \mathrm{e}^{k T_{i}}-\frac{\mathrm{e}^{-(\lambda+\gamma)(t-14)}}{\lambda+\gamma} \sum_{i=0}^{T-14} \mathrm{e}^{(\lambda+\gamma) i}\right]+E .
\end{aligned}
$$

Using the initial condition from (A2), we obtain the solution

$$
\begin{aligned}
x_{2}(t)= & \frac{\gamma \delta k}{\lambda+\gamma-k}\left[\sum_{i=0}^{T}\left(\frac{-\mathrm{e}^{-k(t-i)}}{k}+\frac{\mathrm{e}^{-(\lambda+\gamma)(t-i)}}{\lambda+\gamma}\right)\right. \\
& \left.+\sum_{i=0}^{T-14}\left(\frac{\mathrm{e}^{-k(t-14-i)}}{k}-\frac{\mathrm{e}^{-(\lambda+\gamma)(t-14-i)}}{\lambda+\gamma}\right)+\frac{14}{k}-\frac{14}{\lambda+\gamma}\right] .
\end{aligned}
$$




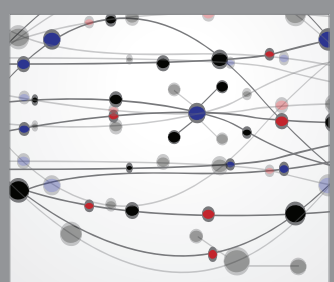

The Scientific World Journal
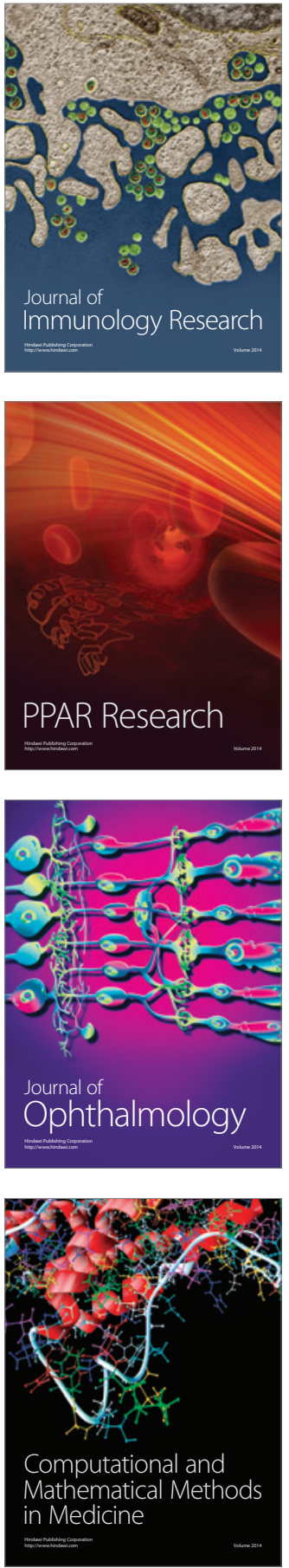

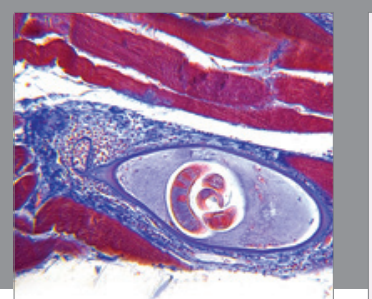

Gastroenterology Research and Practice

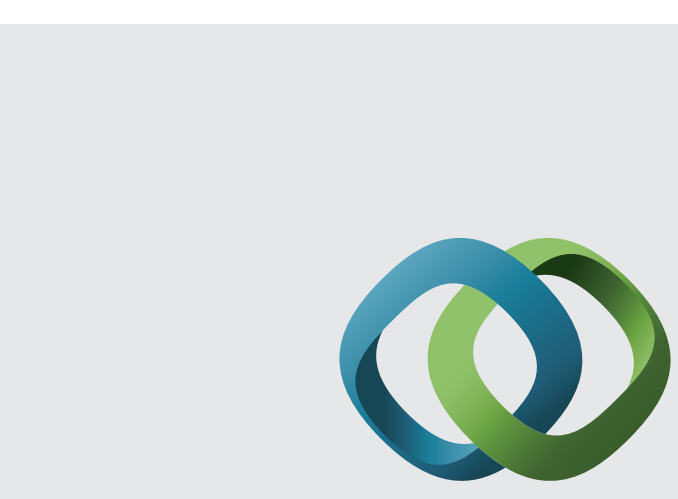

\section{Hindawi}

Submit your manuscripts at

http://www.hindawi.com
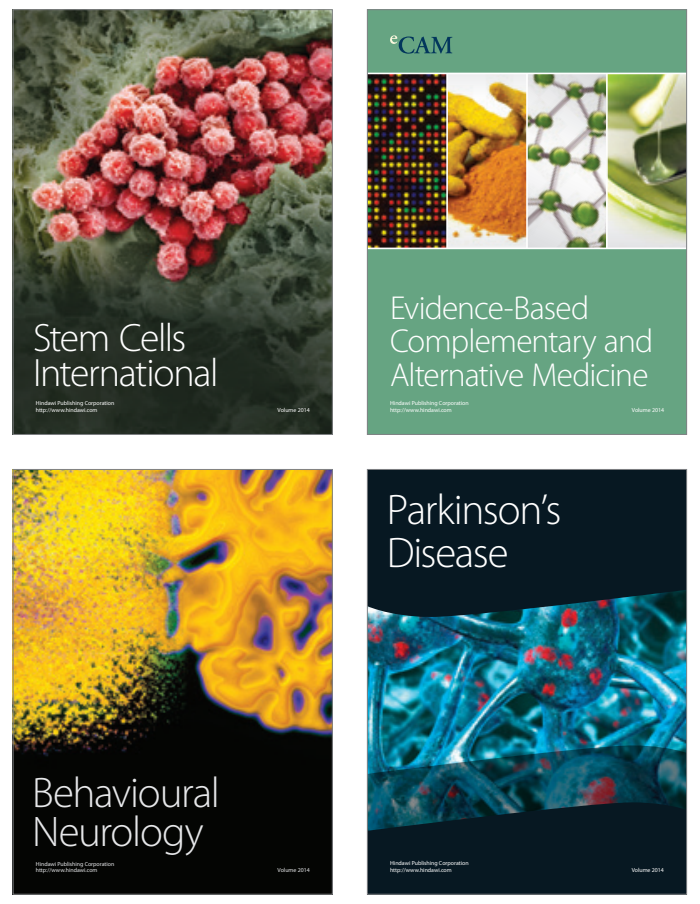
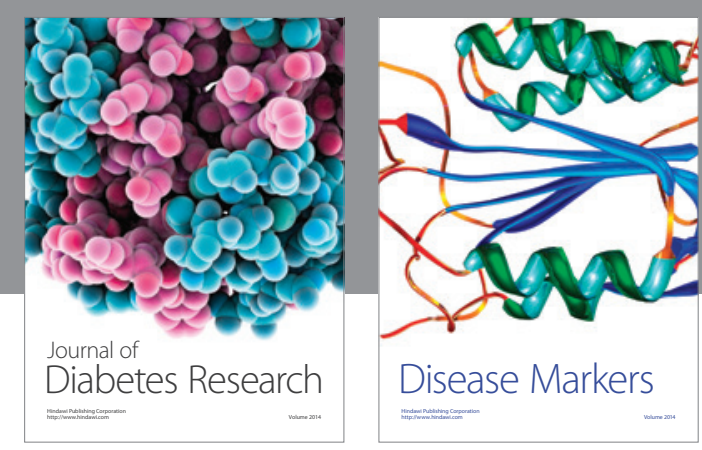

Disease Markers
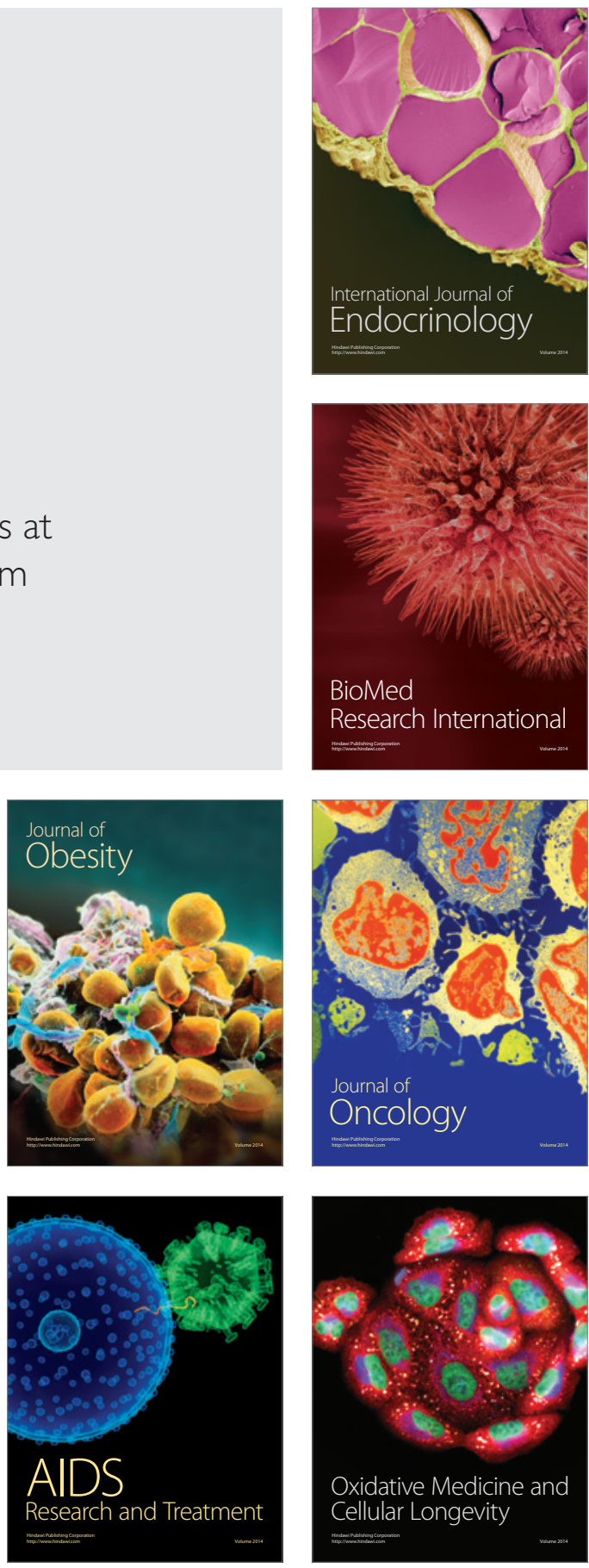